\title{
The Value of Laparoscopic Total Mesorectal Excision and Circumferential Resection Margin in the Treatment of Distal Rectal Cancer: Single Center Experience
}

\author{
Samy Abbas Elbaz ${ }^{*}$ Waleed Hassan Omar, Hosam Ghazy Elbanna, Mohamed Youssef, \\ Sameh Hany Emile, Mohamed Farid \\ General Surgery Department, Colorectal Surgery Unit, Mansoura University Hospital, Mansoura University, Mansoura City, Egypt \\ Email address: \\ Sami_mrcsed@yahoo.com (S. A. Elbaz), Waleed_h_omar@yahoo.com (W. H. Omar), Hosamelbanna@hotmail.com (H. G. Elbanna), \\ M_elkhir@yahoo.com (M. Youssef), Sameh200@hotmail.com (S. H. Emile), Mfshzk2005@yahoo.com (M. Farid) \\ ${ }^{*}$ Corresponding author
}

\section{To cite this article:}

Samy Abbas Elbaz, Waleed Hassan Omar, Hosam Ghazy Elbanna, Mohamed Youssef, Sameh Hany Emile, Mohamed Farid. The Value of Laparoscopic Total Mesorectal Excision and Circumferential Resection Margin in the Treatment of Distal Rectal Cancer: Single Center Experience. Journal of Surgery. Vol. 4, No. 5, 2016, pp. 114-121. doi: 10.11648/j.js.20160405.15

Received: August 22, 2016; Accepted: August 30, 2016; Published: September 18, 2016

\begin{abstract}
Total mesorectal excision (TME) has emerged as a method for complete cure of rectal cancer with promising results. The present study aimed to evaluate the technical feasibility and the clinical and oncological outcomes of laparoscopic TME with abdominoperineal resection (APR) for distal rectal carcinoma. Twenty patients with distal rectal carcinoma were treated with laparoscopic APR and TME in the period of January 2012 to March 2015. Patients' demographics, clinical symptoms, operation time, complications, pathological characteristics of the rectal tumor, and the local and distant recurrence of the tumor were recorded and analyzed. The study included 11 (55\%) female and 9 (45\%) male of a mean age of $46.9 \pm 10.8$ years. The mean distance of the tumor from the anal verge was $3.35 \pm 0.9 \mathrm{~cm}$. The mean operation time was $182 \pm 7$ minutes. Adenocarcinoma accounted for $55 \%$ of cases, whereas mucinous adenocarcinoma was detected in $40 \%$ of patients, and signet ring carcinoma in $5 \%$. The mean circumferential resection margin (CRM) was $4.6 \pm 3.5 \mathrm{~mm}$. The mean duration of hospital stay was $9.21 \pm 6.9$ days. Perioperative complications were recorded in seven patients $(35 \%)$. Five $(25 \%)$ cases were converted to open surgery. The median follow-up duration was 18 months. Local recurrence was diagnosed in two (10\%) cases. Laparoscopic TME is a technically feasible procedure, yet requires adequate training and sufficient knowledge of the anatomy of the pelvis. Although all patients underwent APR and $90 \%$ of them received neoadjuvant treatment; the local recurrence was still higher than other studies which can be attributed to the pathologic characters and the stage of the tumors.
\end{abstract}

Keywords: Laparoscopic, TME, Abdominoperineal Resection, Rectal Cancer, CRM, Outcome

\section{Introduction}

The treatment of rectal cancer has been thoroughly discussed in a considerable number of published studies [1-4] with a remarkable evolution in the technical aspects and the outcomes achieved. Regardless the operative technique employed; the ultimate goal is to accomplish complete clinical and pathological cure [5] together with satisfactory functional outcomes.

In the modern era, all efforts in rectal cancer surgery have been directed towards two essential objectives: ensuring radical eradication of the tumor, and maintaining an acceptable level of life quality by avoiding permanent stoma [4]. This has been clearly reflected in the increasing use of anal sphincter preserving procedures and the routine performance of total mesorectal excision (TME) whether through an abdominal or transanal approach.

In order to elucidate the definition of TME, some anatomic concepts need to be clarified. The mesorectum is not a true mesentery but rather a fatty tissue that envelops the rectum and in which the blood and lymphatic vessels, lymph nodes and autonomic nerves are carried. From this anatomic perspective we can expect that localized rectal cancer 
remains confined to the mesorectal plane, thus, complete removal of this package would guarantee the radical cure for the tumor [6].

In addition to the complete eradication of the tumor, the other virtue of TME is the preservation of the autonomic nerves which help maintains the sexual function. Nevertheless, TME performed in the setting of abdominoperineal resection (APR) is still associated with a higher risk of sexual impotence [7]. APR is not only associated with higher rates of impotence, but is also associated with a higher positive circumferential margin (CRM) that can reach up to three folds that of low anterior resection [8].

Despite its advantages, TME is considered a difficult technique owing to the complicated anatomy and the narrow spaces of the pelvis. The exact identification of the proper "holy" plane can be challenging and inadvertent dissection in the wrong plane is possible with the inexperienced hands. Therefore, sufficient training and adequate knowledge of the relevant anatomy of the operative field are required since mastering this technique warrants a steep learning curve [6].

The majority of the published trials reported the outcome of laparoscopic TME performed with sphincter-saving low or ultra-low anterior resection. However, the present study attempted to evaluate the technical feasibility as well as the clinical and oncological outcomes of laparoscopic TME in the setting of abdominoperineal resection for low-seated rectal carcinoma.

\section{Patients and Methods}

\subsection{Study Design and Setting}

This is a prospective case series study on 20 patients with distal rectal carcinoma who were admitted to the colorectal surgery unit and general surgery department of Mansoura University hospitals and treated with laparoscopic-assisted abdominoperineal resection and TME in the period of January 2012 to March 2015. Ethical approval of the study protocol was obtained from the board of general surgery department of Mansoura faculty of medicine.

\subsection{Inclusion/Exclusion Criteria}

Patients included in the study had non-metastatic resectable rectal cancer. Patients with bulky tumors $(>6 \mathrm{~cm}$ on pelvic CT), complicated tumors (perforation, hemorrhage and intestinal obstruction), pregnant patients, and patients with contraindications to laparoscopy or unfit for general anesthesia were excluded.

\subsection{Preoperative Assessment}

Full history was taken from all patients including demographic data, present complaint, past history of surgical intervention and family history of similar or related conditions. General and abdominal examinations were conducted to exclude the presence of complicated tumors, or signs of metastasis.
Local digital examination was performed to assess the tumor regarding its size, mobility, location, relation to the sphincter mechanism and to the anorectal ring, and distance from the anal verge. In addition, complete pelvic examination was done in female patents to determine vaginal invasion or spread to the ovaries. Rigid proctoscopy was done in seven cases to demonstrate the proximal and distal levels of the mass from the anal verge and the extent of circumferential involvement and to obtain adequate tissue biopsy.

Patients were subjected to routine laboratory investigations as complete blood count, liver and kidney function tests and prothrombin time, and tumor markers as carcinoembryonic antigen. Pelvi-abdominal ultrasound was performed for the initial assessment to exclude hepatic focal lesions or ascites. Pelvi-abdominal CT with oral and I.V contrast or MRI were done for staging of the tumor by demonstrating regional tumor extension, lymphatic and distant metastases, and tumor related complications such as perforation or fistula formation. Barium enema was used to help in diagnosis, localization of the tumor, and to exclude other colonic lesions. In addition, metastatic workup was done routinely for all patients to exclude distant metastasis. Colonoscopy was conducted in all patients to confirm the diagnosis, exclude other lesions, and take 4-8 quadrant biopsies.

\subsection{Operation}

\subsubsection{Preparation}

Standard bowel preparation was conducted with restriction of oral intake to clear liquid diet for 48 hours prior to the operation, and rectal enemas at the night of the operation. One gram of cefotaxime was administered intravenously at the night of surgery as prophylactic systemic antibiotic. The day before operation patients were given prophylactic subcutaneous low molecular weight heparin.

Written informed consents were taken from all patients explaining the nature of the disease, details of surgery, advantages of minimally invasive approach, clarifying the possible complications of surgery and the possibility of conversion to open surgery.

\subsubsection{Operative Technique}

All procedures were performed under general anesthesia with patients in the Lioyd Davies Trendelenburg position. One gram of third generation cephalosporin and 500mg of metronidazole were given with induction of anaesthesia.

Four to five ports technique was used. The first cannula was positioned in the umbilical region either above or below the umbilicus. We inserted $10-\mathrm{mm}$ and $5-\mathrm{mm}$ trocars in the right lower quadrant for the dissection by the operating surgeon, and at least one additional 5-mm port in the left lower quadrant for the assistant to provide retraction. Additional 5th cannula was inserted in the suprapubic area in the midline 1-2 fingers above the pubis.

The entire peritoneal cavity was carefully explored, then the dissection started by retracting the mesosigmoid in a ventrolateral direction to help expose the inferior mesenteric artery (IMA), then the peritoneum was incised down to the 
right of the superior rectal artery starting at the sacral promontory (figure 1).

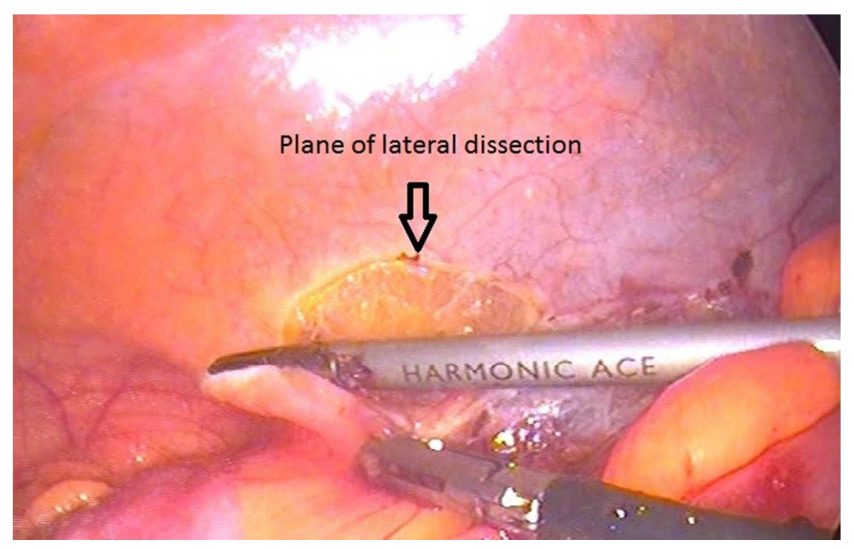

Figure 1. Start of laparoscopic lateral dissection.

Under continuous traction, the peritoneum was incised cephalad towards the origin of the IMA. Using a combination of gentle spreading and electrosurgical dissection, the IMA was swept ventrally and the preaortic hypogastric neural plexus was swept dorsally to prevent injury, then the IMA was divided using ultrasecision (Harmonic ace) device or endoscopic stapler (figure 2).

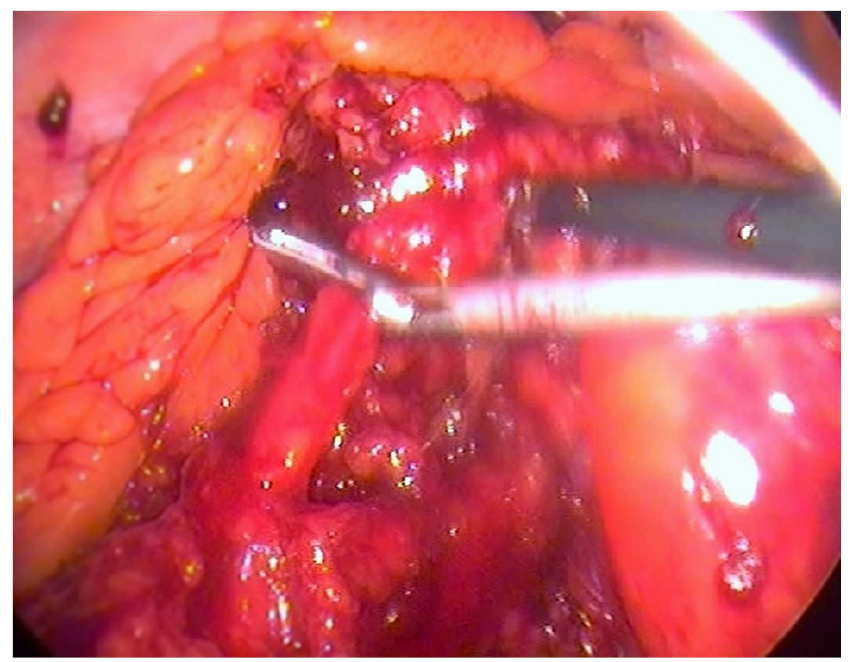

Figure 2. High ligation of IMA by Harmonic ace scalpel.

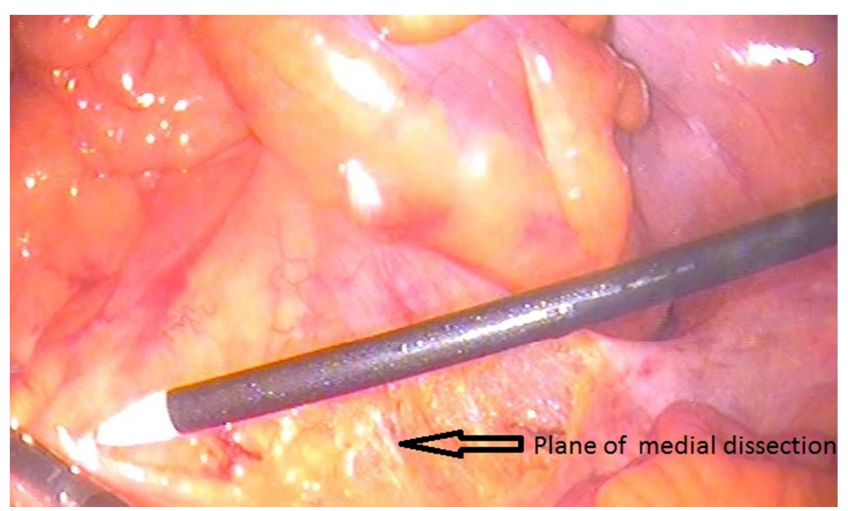

Figure 3. Medial dissection to identify the ureter.
Dissection then was continued medially beneath the artery (figure 3), the left ureter and gonadal vessels were identified and swept posteriorly. With the IMA identified and ligated, the peritoneum was incised anteriorly over the pedicle, dissecting leftward toward the inferior mesenteric vein (IMV). Careful dissection was used to create a peritoneal window just lateral to the IMA and IMV. This pedicle is ligated high above the left colic artery using a harmonic scalpel device leaving a stump of $1-1.5 \mathrm{~cm}$ long.

The sigmoid colon was completely mobilized using a sharp and blunt dissection. The proximal resection line just to the left of the inferior mesenteric pedicle was held using "triangulating tension," and was transected up to the proximal intestinal resection line by the LigaSure device. The colon was divided with a cartridge of a 45 or $55 \mathrm{~mm}$ endoscopic stapler.

The rectum was completely mobilized down to the pelvic floor following the standard TME surgical principles. The dissection is commenced with posterior mobilization, working between the fascia propria of the rectum and the presacral fascia, initially dissecting sharply using the LigaSure device, or harmonic scalpel, as far distally as possible. The dissection was continued posterolaterally to the right and left sides of the rectum, carefully and continuously sweeping the hypogastric nerves trunks posteriorly and laterally. This was continued until the tip of the coccyx was reached. The lateral stalks were divided using the LigaSure device, taking care to separate the pelvic nerve plexus from the rectum at the level of the lateral stalks.

The anterior plane, at the pelvic cul-de-sac, is struck usually after most of the posterior and lateral dissection has been completed. The first assistant uses the left hand to retract the anterior portion of the reflection anteriorly, and the right hand to retract the rectum superiorly and posteriorly, whereas the surgeon uses the left hand to retract the rectum medially (for the right side of the dissection) and the right hand is dividing tissue using the LigaSure.

Upon completion the abdominal part of the procedure, the $\mathrm{CO}^{2}$ pneumoperitoneum was maintained to help alert the surgeon to the proper plane as the perineal dissection proceeded. The anus was occluded with a silk 0 purse-string suture, then the perineal dissection was performed starting posteriorly then laterally and finally anteriorly using an elliptical incision. The pelvic cavity was entered posteriorly initially, with release of the pneumoperitoneum, then perineal excision of the anus and rectum comprising the sphincter complex was done. The specimen was extracted and irrigation was accomplished from below. Finally, a drain was placed in the pelvis.

The perineal wound was then closed in layers over a suction drain. The specimen (figure 4) was opened, inspected, and measured in the operating room. The predetermined colostomy site was prepared and terminal colostomy was created and matured using 3/0 Vicryl suture. The abdominal cavity and the pelvis were carefully assessed laparoscopically for haemorrhage, then the trocar sites were closed, and occlusive dressings were placed over them. 


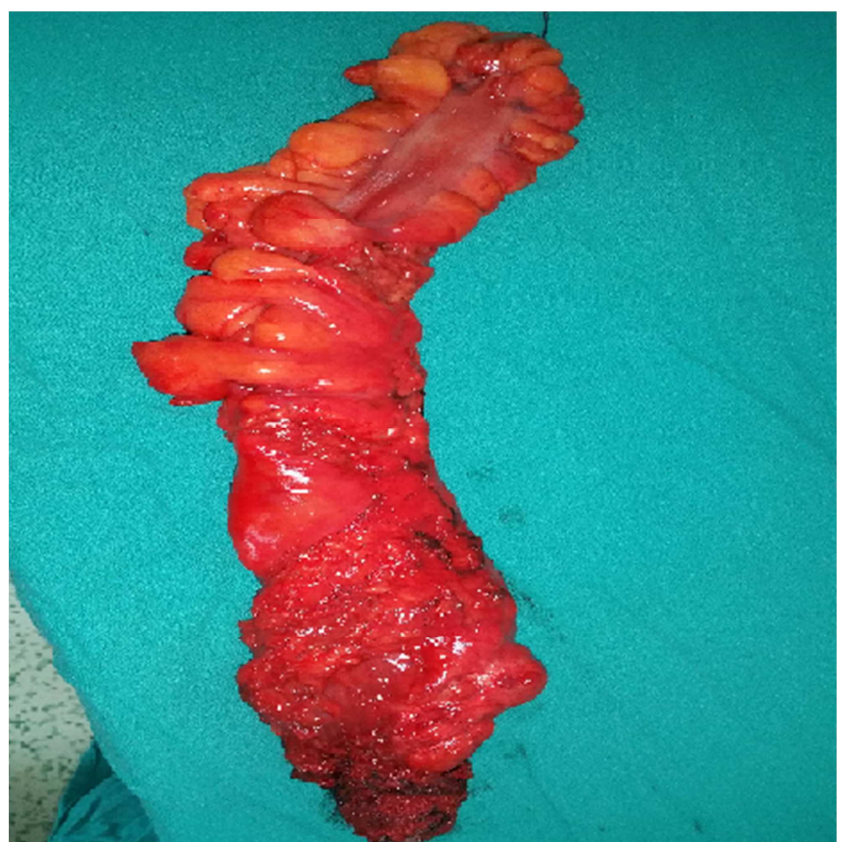

Figure 4. Resected specimen.

\subsubsection{Postoperative Care}

Patients were transferred after the operation to the recovery room and then to the normal unit except if there was any indication for ICU admission. Analgesia was achieved via intravenous Nalufin $10 \mathrm{mg}$ which was repeated every 6 hours when necessary.

Enhanced recovery program was implemented including early oral intake, early mobilization. The colostomy was observed for viability and functioning. Pelvic drain was usually removed by the 3rd day. Urinary catheter was usually removed 7 to 10 days after the procedure. If the postoperative course was uneventful, patients were discharged on the 4 th to the 6th postoperative day.

\subsection{Follow Up}

Patients were followed every 3 months in the outpatient clinic for the first two years, and then every 6 months. During such visits, history taking and physical examination were done, and blood samples were obtained to check CEA. Pelvi-abdominal CT scan was done routinely for all patients at 12 months of follow-up to exclude recurrence of the tumor. On suspicion of recurrence, further investigations including colonoscopy and biopsy were ordered.

\subsection{Outcomes Assessed}

\section{Short term outcomes}

The operation time, times of 1 st bowel motion and 1 st passing flatus, postoperative ambulation, duration of hospital stay, and postoperative morbidity and mortality were recorded. Conversion was defined as the inability to complete dissection, including vascular pedicle, via laparoscopy and the need for making an abdominal prematurely. The necessity for an abdominal incision to deal with any intra-abdominal complication was also considered conversion. Also, pathological outcomes as the length of resection safety margin, and the number of harvested lymph nodes were assessed

Long term outcomes

Long-term outcomes comprised the duration of follow-up, incidence and pattern of recurrence, recurrence-free survival, and overall mortality rate.

\subsection{Statistical Analysis}

Data were analyzed using Excel and SPSS version 21 under Microsoft windows (Bristol, UK). Comparison of the categorical variables was performed using fisher's exact test or Chi square test where appropriate. Continuous variable were compared using Student t-test when variables were normally distributed. Analysis of the risk factors for conversion to open procedure was performed using Cox regression test. $P$ values of less than 0.05 were regarded statistically significant.

\section{Results}

\subsection{Patients' Characteristics}

The study was conducted on 20 patients with distal rectal cancer who underwent laparoscopic abdominoperineal resection with TME. Patients were 11 (55\%) female and 9 (45\%) male of a mean age of $46.9 \pm 10.8$ (range, 23-68) years. $60 \%$ of the patients aged below 60 years. The mean BMI of patients was $26.4 \pm 5.9$ (range, $18-40$ ) $\mathrm{kg} / \mathrm{m}^{2} .55 \%$ of patients had normal BMI, 35\% had a BMI over 25, and $10 \%$ had BMI below 25 .

The commonest presenting symptom was rectal bleeding in 14 patients $(70 \%)$, followed by mucus discharge in 9 (45\%) patients. Five $(25 \%)$ patients presented with progressive constipation, and three (15\%) patients complained of rectal or anal pain.

By clinical examination, all patients had palpable tumor in the lower third of the rectum (within $6 \mathrm{~cm}$ from anal verge). The mean distance of the tumor from the anal verge was 3.35 \pm 0.9 (range, $1-6$ ) cm. $60 \%$ of patients had a tumor within 3-4 $\mathrm{cm}$ from the anal margin. Eighteen $(90 \%)$ patients completed a preoperative course of neoadjuvant radiotherapy whereas two $(10 \%)$ patients refused to have neoadjuvant therapy prior to the procedure.

\subsection{Technical Details}

The mean length of the operation calculated from the time of insertion of the first trocar till the end of laparoscopic part of procedure was $182 \pm 7$ (range, 120-240) minutes. The operative time for half of the patients was between 120-180 minutes. Intra-operative blood loss was estimated by calibrated suction device. The mean blood loss was $537.5 \pm$ 199 cc ranging from $200-1000 \mathrm{cc}$.

\subsection{Pathological Characteristics of the Tumor}

The length of the resected specimen was measured 
postoperatively from the perineal skin distally to the proximal resection margin. The mean length of the specimen was 25.3 (range, 18-38) $\mathrm{cm}$. The largest diameter of the resected tumor was measured by a ruler, it ranged from 2$6 \mathrm{~cm}$ with a mean of $4.5 \pm 0.6 \mathrm{~cm}$.

Naked eye examination of resected specimen reveled six (30\%) malignant ulcers, six (30\%) annular stenosing lesions, and eight (40\%) cauliflower masses.

Microscopic examination revealed that adenocarcinoma accounted for $55 \%$ of cases, whereas mucinous adenocarcinoma was detected in $40 \%$ of patients, and signet ring carcinoma in 5\%. Adenocarcinoma was well differentiated, moderately differentiated, and poorly differentiated in six, four, and one patient, respectively.

Regarding the pathologic staging of the tumor, $50 \%$ of patients had T3 staging, whereas $25 \%$ had $\mathrm{T} 4$, and the remaining $25 \%$ were of stage T1-2. According to the AJCC staging, $55 \%$ of the tumors were of stage III while $30 \%$ were of stage II, and $15 \%$ were of stage I.

The number of harvested lymph nodes is shown in table (1). In nine patients, all harvested LNs were negative, whereas in 11 patients there were positive LNs infiltrated with malignancy. Number of positive LNs is shown in table (2).

Table (1). Distribution of the patients according to the number of harvested lymph nodes.

\begin{tabular}{ll}
\hline Total LN harvested & No of patients (\%) \\
\hline 1 & $5(25)$ \\
$2-4$ & $4(20)$ \\
$5-8$ & $7(35)$ \\
$9-12$ & $1(5)$ \\
$13-16$ & $3(15)$ \\
\hline
\end{tabular}

Table (2). Number of negative and positive lymph nodes in the studied group.

\begin{tabular}{ll}
\hline LN infiltrated with malignant metastasis & Number (\%) \\
\hline Negative $L N$ & $9(45)$ \\
Positive $L N$ & $11(55)$ \\
Single LN [N1a] & $5(25)$ \\
Two - three [N1b] & $4(20)$ \\
Four - six [N2a] & $1(5)$ \\
Seven and more [N2b] & $1(5)$ \\
\hline
\end{tabular}

The CRM was assessed microscopically by the pathologist; the mean CRM was $4.6 \pm 3.5$ (range, 0-12) $\mathrm{mm}$. A CRM of 1 or $2 \mathrm{~mm}$ was considered free or none infiltrated. Distribution of the patients according to the examined CRM is shown in table (3). Proximal and distal cut ends were also examined by the same pathologist and all were free from any tumor growth.

Table (3). Distribution of the patients studied according to the circumferential resection margin (CRM).

\begin{tabular}{ll}
\hline CRM & No of patients (\%) \\
\hline Zero & $2(10)$ \\
$1-2$ & $4(20)$ \\
$3-6$ & $9(45)$ \\
$7-12$ & $5(25)$ \\
\hline
\end{tabular}

\subsection{Postoperative Data}

Patients required postoperative analgesia for a mean period of $4.91 \pm 2.7$ days, patients passed flatus after a mean period of $3.5 \pm 2.5$ days, and passed the first motion after $4.6 \pm 2.1$ days. The mean duration for patients to resume full oral diet was $4 \pm 1.4$ days. The mean duration of hospital stay was $9.21 \pm 6.9$ days.

No operative mortality was recorded in the study. Seven patients $(35 \%)$ developed 14 various complications. There were four (20\%) major complications and $10(50 \%)$ minor complications as illustrated in table (4). Five (25\%) cases were converted to open surgery; risk factors for conversion were analyzed and displayed in table number (5).

Table (4). Operative morbidities detected in the patients studied.

\begin{tabular}{l|l}
\hline Morbidity & No (\%) \\
\hline Major morbidity & $1(5)$ \\
Injury of left ureter & $1(5)$ \\
Intraoperative bleeding & $1(5)$ \\
Small bowel injury & $1(5)$ \\
Pulmonary embolism & \\
Minor morbidity & $2(10)$ \\
Perineal Wound infection & $1(5)$ \\
Colostomy retraction & $1(5)$ \\
Acute pneumonia & $2(10)$ \\
Urinary retention & $4(20)$ \\
Urinary tract infection & \\
\hline
\end{tabular}

Table (5). Cox regression analysis of risk factors for conversion to open procedure.

\begin{tabular}{llll}
\hline Variable & $\begin{array}{l}\text { Conversion } \\
(\mathbf{n = 5})\end{array}$ & $\begin{array}{l}\text { Non conversion } \\
(\mathbf{n = 1 5})\end{array}$ & P value \\
\hline Age & $49.2 \pm 11.3$ & $44.5 \pm 9.8$ & 0.38 \\
Gender (M/F) & $3 / 2$ & $6 / 9$ & 0.61 \\
BMI & $29.4 \pm 5.8$ & $26.2 \pm 5.6$ & 0.28 \\
Operative time & $202 \pm 8.2$ & $180.6 \pm 7.7$ & $<0.0001$ \\
$\begin{array}{l}\text { Intraoperative } \\
\text { complications (n/total) }\end{array}$ & $2 / 5$ & $1 / 15$ & 0.14 \\
\hline
\end{tabular}

The median follow-up duration was 18 (range, 12-36) months. Local recurrence was diagnosed in two (10\%) cases, whereas no distant metastasis was detected. Both patients who had local recurrence were male aging 28 and 46 years, with positive CRM and had a stage III rectal mucinous carcinoma. One of the patients who developed recurrence received neoadjuvant therapy while the other did not. Recurrence occurred at nine and 12 months after the procedure, respectively.

\section{Discussion}

Heald first described TME for rectal cancer surgery in 1979 [9], he devised sharp under vision dissection of the mesorectum in an avascular plane which allows the preservation of the autonomic nerves [10]. Thirteen years later, MacFarlane et al. [11] published their experience with TME reporting local recurrence in $4 \%$ of patients only. Enker et al. [12] and Moriya et al. [13] reported higher recurrence rates of $7.4 \%$ and $9.3 \%$, respectively after TME with 
extensive lymphadenectomy.

Since TME has been deemed the new standard treatment for rectal cancer, the completeness of the TME and the quality of the specimen were considered the major predictors of success and long-term survival. The completeness of TME was classified into complete, near complete, and inadequate. Complete TME was defined as a "complete removal of the lymph node bearing mesorectum along with its intact enveloping fascia" [14].

The components of complete TME are the high ligation of the IMA, full mobilization of the splenic flexure, division of the colon at the descending sigmoid junction, sharp dissection in the avascular plane into the pelvis anterior the presacral fascia and outside the fascia propria or enveloping visceral fascia, division of lymphatic and middle hemorrhoidal vessels anterolaterally, and inclusion of all pelvic fat and lymphatic material at least $2 \mathrm{~cm}$ below the level of the distal margin [15].

TME can be achieved via transabdominal or transanal routes, the transabdominal technique can be conducted through an open, laparoscopic or robotic approach. Recently, the attention has been diverted to the transanal TME (TaTME) owing to its advantages that include enhanced visualization of the nerve bundles and the distal margin of the tumor, and easier anterior dissection in male patients. A recent systematic review involving 510 patients with rectal cancer who underwent TaTME reported only one mortality and a morbidity rate of $35 \%$ with anastomotic leakage occurring in $6.1 \%$ of the patients. TME was complete in $88 \%$ of the cases, nearly complete in $6 \%$ and incomplete in $6 \%$. The CRM was negative in $95 \%$ of cases and the distal resection margin was negative in $99.7 \%$ [16].

Despite the marvelous results of TaTME; the laparoscopic TME still has its place in the current time since TaTME has been described as a technically demanding procedure that requires designated surgical instruments and adequate training and education before mastering the technique. Hence, the present study aimed at evaluating the technical feasibility and outcomes of the laparoscopic TME in patients with distal rectal cancer who underwent abdominoperineal resection.

The laparoscopic TME entails some technical aspects that need to be elaborated. First, unlike the open approach, medial to lateral dissection is preferred as it facilitates the splenic mobilization; also the division of the IMA first opens up the dissection proceeding superiorly along the base of left colon mesentery to the IMV and splenic flexure [17]. After the IMA has been divided, the posterior plane is identified and entered. The division of the gutter of the right mesorectum below the IMA at the pelvic brim can open up the posterior plane anterior to the presacral fascia to facilitate identification of structures at the pelvic brim [14].

Laparoscopy makes the identification of the ureter easier due to the magnification of the camera and close visualization of the sidewall. The medial to lateral approach to the identification of the ureter confers shorter operative time, and less rate of conversion than the lateral approach.
However, the lateral approach is still employed in the case of large pelvic masses or altered anatomy from inflammation [18].

Another advantage of laparoscopic approach over the open counterpart is the better visualization of the sacral nerves not only at the pelvic brim but also in the deep lateral pelvic floor by the magnified camera. Despite the improved visualization of the nerves at the sacral promontory, nerve dysfunction after laparoscopic procedures was still comparable with open procedures [14]. The medial to lateral approach followed in the laparoscopic approach helps identify the IMA in an easier and faster way. However, the increasing use of energy sealing devices can pose some risk on the adequate sealing of the IMA. In addition, if the bowel was not properly retracted an injury to the arcade vessel in the left colon can jeopardize the blood supply of the proximal bowel.

Although the laparoscopic approach offers tangible benefits compared to the open approach; the difficult pelvis can be still a challenging problem to the surgeon. Obesity, male gender, and previous surgery are risk factors for intraoperative injuries, incomplete TME, positive CRM, and failed anastomosis [19].

We studied 20 patients with distal rectal carcinoma who underwent laparoscopic TME. Around one-third of the patients were classified as overweight or obese (BMI $>25$ ). Obesity, as previously stated, is a risk factor for technical failures and complications. Difficulties we encountered when operating upon the obese patients involved the increased adipose tissue of the mesentery and peritoneum which reduced peritoneal space and led to foreshortening of the colonic mesentery making it harder to retract the small intestine and to completely mobilize the splenic flexure. Visceral obesity also concealed the vasculature and the nerves making their identification difficult [20].

The mean age of the patients was 47 years, with half of the patients aging between 40 and 60 years, this renders our patients younger than what the Surveillance, epidemiology, and end results program (SEER) cancer statistics reported that more than $90 \%$ of colorectal cancers are detected in patients aging above 50 years [21]. Patients had an almost equal gender distribution coping with the literature that reported similar incidence of colorectal cancer in both sexes [22].

The pathological characteristics of rectal tumors in the studied group were unique. $80 \%$ of the neoplasms were located four or less centimeters from the anal verge, less than the reported distance of rectal tumors from the anal verge (6 $\mathrm{cm}$ by Morino et al. [23] and $9 \mathrm{~cm}$ by Leroy et al. [24]. This close proximity to the anal verge hindered the performance of sphincter-preserving procedures and was an indication for abdominoperineal resection in all patients.

Another factor that supported the decision for APR was the pathological type of the tumor. Half of the patients had an aggressive type of rectal carcinoma with typically poor prognosis. It was notable that $40 \%$ of patients had mucinous carcinoma which is higher than the usual incidence of $5-10 \%$ of all rectal cancers [25]. Mucinous carcinoma is known to 
be associated with higher incidence of CRM positivity, greater lymph node affection, and higher incidence of peritoneal and distant spread. Furthermore, mucinous carcinoma shows poor response to neoadjuvant chemoradiation rendering this kind of therapy of questionable benefit in the case of mucinous rectal cancer [26].

Three-quarter of the patients had a locally advanced disease, with infiltration of the anal sphincters, thus, the decision for APR in these patients was justified and rational. The mean number of harvested lymph nodes was around five, less than what Leroy and colleagues [24] reported (8 lymph nodes). In more than half of the patients in our study the harvested lymph nodes were infiltrated by malignancy acquiring stage III by the TNM classification system.

Two patients had an infiltrated CRM, the CRM status can be used as a predictor of survival and tumor recurrence after rectal cancer surgery and is considered a useful indicator of the quality of surgery. Birbeck and colleagues [27] conducted a study on 586 patients with rectal cancer, the CRM positivity rate was around $28 \%$. Patients with negative CRM had both better survival and lower recurrence rates $(10 \%$ vs $38 \%$ ) in comparison with patients with infiltrated CRM. This was confirmed by the present study as both patients who developed local recurrence had positive CRM. The incidence of positive CRM in the present trial $(10 \%)$ is close to what was reported by van Leeresum and colleagues [28] that the rates of positive CRM after APR and low anterior resection were $12 \%$ and $8 \%$, respectively with no significant differences observed between the two procedures.

The mean operation time (182 minutes) was higher than what Yang et al. [29] reported, yet lower than the operation time reported by other investigators [23, 24]. The operation time was close to four hours in three patients in whom intraoperative complications occurred. Inadvertent injury of the small bowel occurred in one patient, intraoperative hemorrhage secondary to vessel injury in another patient, and injury to the left ureter in the third patient. Overall, around one-third of the patients developed surgery-related morbidities with only four major complications. The complication rate in the current study is comparable to what Morino and colleagues [23] reported, yet higher than the morbidity rate $(27 \%)$ disclosed by Leroy et al [24].

Five laparoscopic procedures were converted to open procedures either due to the occurrence of intraoperative complications, or due to prolonged operation time with difficult dissection and/or visualization of anatomic structures. The conversion rate was twice that in a previous report [23], and much higher than the reported incidence by Yang et al. [29]. The regression analysis we performed demonstrated that prolonged operation time beyond 180 minutes with failure to progress through the operative steps was the only significant factor that predicted conversion to open procedures.

Local recurrence of rectal carcinoma occurred in two patients; both were males and were detected within the first year postoperatively. It was notable that both cases had a positive CRM, highlighting the clinical implication of this important pathologic parameter. Leroy and colleagues [24] reported lower rate of local recurrence $(6 \%)$ after a relatively longer duration (16 months). Furthermore, Morino et al. [23] reported a locoregional pelvic recurrence rate of $4.2 \%$. The higher incidence of local recurrence in the present trial can be attributed to the pathologic characters of the tumors since both patients had mucinous adenocarcinoma and stage III by the TNM staging system.

Although the present study shed light on the feasibility and outcomes of laparoscopic TME in the setting of APR for locally advanced distal rectal tumors; the study was limited by the small number of patients studied and the relatively short follow-up duration. We recommend conducting further long-term trials on the feasibility and outcome of laparoscopic TME in comparison with TaTME with abdominoperineal resection for distal rectal carcinoma.

\section{Conclusion}

In conclusion, laparoscopic TME is a technically feasible procedure, yet requires adequate training and sufficient knowledge of the anatomy of the pelvic region. The characteristics of the rectal malignancies in this study had a substantial impact on the clinical decision-making strategy and the ultimate outcome. Although all cases underwent APR and $90 \%$ of them received neoadjuvant treatment; the local recurrence was still higher than other studies which can be attributed to the pathologic characters and the stage of the tumors.

\section{References}

[1] Otani T, Isohata N, Kumamoto K, Endo S, Utano K, Nemoto D, Aizawa M, Lefor AK, Togashi K. An evidence-based medicine approach to the laparoscopic treatment of colorectal cancer. Fukushima J Med Sci. 2016 Jul 30.

[2] Negoi I, Hostiuc S, Paun S, Negoi RI, Beuran M. Extralevator vs conventional abdominoperineal resection for rectal cancerA systematic review and meta-analysis. Am J Surg. 2016 May 11. doi: 10. 1016/j. amjsurg. 2016. 02. 022.

[3] Helewa RM, Park J. Surgery for Locally Advanced T4 Rectal Cancer: Strategies and Techniques. Clin Colon Rectal Surg. 2016 Jun; 29 (2): 106-13. doi: 10. 1055/s-0036-1580722. Review.

[4] Mulsow J, Winter DC. Sphincter preservation for distal rectal cancer--a goal worth achieving at all costs? World J Gastroenterol. 2011 Feb 21; 17 (7): 855-61. doi: 10. 3748/wjg. v17. i7. 855. Review.

[5] Ryan JE, Warrier SK, Lynch AC, Ramsay RG, Phillips WA, Heriot AG. Predicting pathological complete response to neoadjuvant chemoradiotherapy in locally advanced rectal cancer: a systematic review. Colorectal Dis. 2016 Mar; 18 (3): 234-46. doi: 10. 1111/codi. 13207. Review.

[6] Havenga K, Grossmann I, DeRuiter M, Wiggers T. Definition of total mesorectal excision, including the perineal phase: technical considerations. Dig Dis. 2007; 25 (1): 44-50. 
[7] Havenga K, Enker WE, McDermott K, Cohen AM, Minsky $\mathrm{BD}$, Guillem J: Male and female sexual and urinary function after total mesorectal excision with autonomic nerve preservation for carcinoma of the rectum. J Am Coll Surg 1996; 182: 495-502.

[8] Daniels IR, Strassburg J, Moran BJ: The need for future surgical low rectal cancer studies. Colorectal Dis 2006; 8 (suppl 3): 25-29.

[9] Heald RJ: A new approach to rectal cancer. Br J Hosp Med 1979; 22: 277-281.

[10] Heald RJ, Husband EM, Ryall RD: The mesorectum in rectal cancer surgery - the clue to pelvic recurrence? Br J Surg 1982; 69: 613-616.

[11] MacFarlane JK, Ryall RD, Heald RJ: Mesorectal excision for rectal cancer. Lancet 1993; 341: 457-460.

[12] Enker WE, Thaler HT, Cranor ML, Polyak T: Total mesorectal excision in the operative treatment of carcinoma of the rectum. J Am Coll Surg 1995; 181: 335-346.

[13] Moriya Y, Sugihara K, Akasu T, Fujita S: Importance of extended lymphadenectomy with lateral node dissection for advanced lower rectal cancer. World J Surg 1997; 21: 728732 .

[14] Lichliter WE. Techniques in Total Mesorectal Excision Surgery. Clinics in Colon and Rectal Surgery. 2015; 28 (1): 21-27. doi: 10. 1055/s-0035-1545066.

[15] Trastulli S, Farinella E, Cirocchi R. et al. Robotic resection compared with laparoscopic rectal resection for cancer: systematic review and meta-analysis of short-term outcome. Colorectal Dis. 2012; 14 (4): e134-e156.

[16] Simillis C, Hompes R, Penna M, Rasheed S, Tekkis PP. A systematic review of transanal total mesorectal excision: is this the future of rectal cancer surgery? Colorectal Dis. 2016 Jan; 18 (1): 19-36. doi: 10. 1111/codi. 13151.

[17] Fleshman J W. Philadelphia, PA: Elsevier Saunders; 2013. Laparoscopic low anterior resection.

[18] Ding J, Liao G Q, Xia Y. et al. Medial versus lateral approach in laparoscopic colorectal resection: a systematic review and meta-analysis. World J Surg. 2013; 37 (4): 863-872.

[19] Delaney C P, Pokala N, Senagore A J. et al. Is laparoscopic colectomy applicable to patients with body mass index $>30$ ? A case-matched comparative study with open colectomy. Dis Colon Rectum. 2005; 48 (5): 975-981.
[20] Leroy J, Ananian P, Rubino F, Claudon B, Mutter D, Marescaux J. The impact of obesity on technical feasibility and postoperative outcomes of laparoscopic left colectomy. Ann Surg. 2005; 2013; 241: 69-76.

[21] Ries L AG, Melbert D, Krapcho M, et al. SEER cancer statistics review, 1975-2005. Bethesda, MD: 2008.

[22] Haggar FA, Boushey RP. Colorectal Cancer Epidemiology: Incidence, Mortality, Survival, and Risk Factors. Clinics in Colon and Rectal Surgery. 2009; 22 (4): 191-197. doi: 10. 1055/s-0029-1242458.

[23] Morino M, Parini U, Giraudo G, Salval M, Brachet Contul R, Garrone C. Laparoscopic Total Mesorectal Excision: A Consecutive Series of 100 Patients. Annals of Surgery. 2003; 237 (3): 335-342. doi: 10. 1097/01. SLA. 0000055270. 48242. D2.

[24] Leroy J, Jamali F, Forbes L, Smith M, Rubino F, Mutter D, Marescaux J. Laparoscopic total mesorectal excision (TME) for rectal cancer surgery: long-term outcomes. surg Endosc. 2004 Feb; 18 (2): 281-9.

[25] Symonds DA, Vickery AL. Mucinous carcinoma of the colon and rectum. Cancer 1976; 37: 1891-900.

[26] Simha V, Kapoor R, Gupta R, Bahl A, Nada R. Mucinous adenocarcinoma of the rectum: a poor candidate for neoadjuvant chemoradiation? Journal of Gastrointestinal Oncology. 2014; 5 (4): 276-279. doi: 10. 3978/j. issn. 20786891. 2014. 020.

[27] Birbeck KF, Macklin CP, Tiffin NJ, Parsons W, Dixon MF, Mapstone NP, Abbott CR, Scott N, Finan PJ, Johnston D, Quirke P. Rates of circumferential resection margin involvement vary between surgeons and predict outcomes in rectal cancer surgery. Ann Surg. 2002 Apr; 235 (4): 449-57.

[28] van Leersum N, Martijnse I, den Dulk M, Kolfschoten N, Le Cessie S, van de Velde C, Tollenaar R, Wouters M, Rutten HJ. Differences in circumferential resection margin involvement after abdominoperineal excision and low anterior resection no longer significant. Ann Surg. 2014 Jun; 259 (6): 1150-5. doi: 10. 1097/SLA. 0000000000000225.

[29] Yang Q, Xiu P, Qi X, Yi G, Xu L. Surgical margins and shortterm results of laparoscopic total mesorectal excision for low rectal cancer. JSLS. 2013 Apr-Jun; 17 (2): 212-8. doi: 10. 4293/108680813X13654754534675. 